Received 03/23/2018 Review began 04/02/2018 Review ended 05/27/2018 Published 05/31/2018 Corrected 08/08/2018

(c) Copyright 2018

Lauffer et al. This is an open access article distributed under the terms of the Creative Commons Attribution License CC-BY 3.0 ., which permits unrestricted use, distribution, and reproduction in any medium, provided the original author and source are credited.

\section{Corrected: Pancreatic Cancer: Feasibility and Outcome After Radiochemotherapy with High Dose External Radiotherapy for Non-resected and R1 Resected Patients}

David C. Lauffer ${ }^{1}$, Peter A. Kuhn ${ }^{1}$, Marc Kueng ${ }^{2}$, Sandrine U. Thalmann ${ }^{1}$, Géraldine Risse ${ }^{1}$, Pierre-Alain Tercier $^{1}$, Bernhard Egger ${ }^{3}$, Abdelkarim S. Allal ${ }^{1}$

1. Department of Radiation Oncology, Hospital of Fribourg, Fribourg, CHE 2. Department of Medical Oncology, Hospital of Fribourg, Fribourg, CHE 3. Department of General Surgery, Hospital of Fribourg, Fribourg, CHE

Corresponding author: David C. Lauffer, dc.lauffer@protonmail.com

\section{This article has been corrected.}

Correction date: August 08, 2018. Cite this correction as Lauffer D C, Kuhn P A, Kueng M, et al. (August 08, 2018) Correction: Pancreatic Cancer: Feasibility and Outcome After Radiochemotherapy with High Dose External Radiotherapy for Non-resected and R1 Resected Patients. Cureus 10(8): c14. doi:10.7759/cureus.c14.

Primary and first author David C. Lauffer's affiliation (Hospital of Fribourg) was incorrectly listed as located in Bern, CHE when in fact it is located in Fribourg, CHE. The author's affiliation has been updated to reflect this change.

\section{Abstract}

\section{Background}

Non-resected locally advanced and microscopic positive-margin resected (R1) pancreatic adenocarcinoma are associated with a dismal prognosis. The combination of high dose radiotherapy and concomitant chemotherapy is among the strategies that are used to improve the outcome. The aims of this study were to evaluate the acute and late toxicities and patients' outcome in a retrospective study from a single center.

\section{Material and methods}

From 2009 to 2015, 24 patients, with non-resected locally advanced or R1 resected pancreatic adenocarcinoma, have been treated with concomitant radiochemotherapy, with a median dose of $60 \mathrm{~Gy}$ and gemcitabine (50 mg/m² administered bi-weekly). The acute and late toxicities were evaluated during and after the treatment.

\section{Results}

The actuarial overall survival rates were $39 \%$ at 24 months and $8.6 \%$ at 36 months. The disease-free survival rates were $32.5 \%$ at 24 months and $12.2 \%$ at 36 months. Acute toxicities were mainly grade 1 (G1) to grade 2 (G2) except for one patient who presented with severe digestive bleeding potentially linked to the treatment. Late toxicities consisted mainly of G1 digestive toxicities.

\section{Conclusion}

This study confirms the feasibility of high dose radiotherapy combined with gemcitabine-based chemotherapy in patients with locally advanced pancreatic adenocarcinoma. While the outcome remains unsatisfactory, some patients seem to have benefited from this aggressive therapy, which merits to be investigated further.

Categories: Radiation Oncology, General Surgery, Oncology

Keywords: pancreatic adenocarcinoma, high dose, radiotherapy, survival, toxicities

\section{Introduction}

Pancreatic carcinoma is a rare cancer compared to breast, prostate, and lung cancers. Cancers account for $7 \%$ of all deaths in the US, with pancreatic cancer as the fourth most common cause after lung (26\%), colorectal (8.5\%), and prostate (8\%) cancers for men, and breast (14\%) cancer for women [1-3], making this cancer one of the most deadly. 


\section{Cureus}

The anatomic position of the pancreatic gland in the retroperitoneal space makes it close to many important organs such as stomach, duodenum, and main arteries and veins. Furthermore, pancreatic cancer is characterized by the propensity to progress silently locally and to spread to regional lymphatics. Because of these reasons, at diagnostic, a large percentage of patients (80\% to $85 \%$ ) present an extension to adjacent organs, lymph nodes, fat, soft tissue [4,5], or distant metastases. This makes complete surgical resection difficult, if not impossible [6], with an estimated complete surgical resection of around 10 to $12 \%$ [7].

The only treatment that potentially offers a definitive cure with a long-term survival is a surgical resection with negative margin (R0). In this setting, the reported five-year overall survival rate is about $20 \%$ [8-10]. Either the tumor's extension or the poor performance status (PS) of those patients make the majority of them not operable, and among them only a small part will have a negative surgical margin [11]. A substantial number of patients will have microscopic positive-margin (R1) making their prognosis close to those with unresectable tumors with a five-year overall survival < 5\% [12].

For patients with locally advanced, unresectable non-metastatic disease, multimodal treatments, including radiotherapy and chemotherapy, remain a challenge as for R1 resected patients [13-15]. Several studies, including questionable ones, have tried to establish the role of multimodal approaches, but due to some controversial and conflicting results, the definitive role of these modalities is not yet well established. These treatments have improved only slightly the median survival time to 8-14 months [16-21] despite the improved control of micro-metastasis by the modern systemic therapy regimens [22]. Here we report the results of a combined high dose radiotherapy (RT) with concurrent gemcitabine for locally advanced or R1 resected pancreatic cancers.

\section{Materials And Methods}

Between March 2009 and August 2015, 24 patients (11 females and 13 males) from a single institution (Department of Radiation Oncology at the Hospital of Fribourg, Switzerland) were enrolled in a retrospective study. Eighteen patients had non-resected locally advanced and six had R1 resected pancreatic carcinoma. Histologic confirmation of pancreatic adenocarcinoma was established in all patients. The tumors were considered unresectable either during standard diagnostic workup or after exploratory laparotomy. Four patients presented with cT2-3, 14 with cT4, and six with pT3-4.

Before radiotherapy, all patients underwent a general physical examination. A spiral computed tomography scanning of the upper abdomen in treatment position was performed in all patients. Target delineation was based on data collected and merged from the different imagery procedures and concerned mainly the macroscopic/microscopic areas while elective regional nodes were considered if organs at risk could be well preserved. The mean gross tumour volume (GTV) to clinical target volume (CTV) margin was $5.2 \mathrm{~mm}$ (range: 5-6 mm). The mean CTV to planning target volume 1 (PTV1) margin (tumor + lymph nodes) was $7.4 \mathrm{~mm}$ (range: 5-10 mm). The mean CTV to PTV2 margin (tumor/bed) was $6.3 \mathrm{~mm}$ (range: $5-10 \mathrm{~mm}$ ). All patients received a dose of $59 \mathrm{~Gy}$ or more (median dose $60 \mathrm{~Gy}$ ) except one patient who received $56 \mathrm{~Gy}$. The dose per fraction was mainly between 1.8 and $2 \mathrm{~Gy}$. All patients were treated in supine position; four patients were treated with volumetric modulated arc therapy (VMAT) (TrueBeam, Varian Medical Systems, Inc., CA, USA), 10 patients were treated with intensity modulated radiation therapy (IMRT, TomoTherapy), while the rest were treated with three-dimensional techniques using multiple fields with customized blocks. Special attention was paid to the maximal doses allowed to organs at risk (Table 1). Chemotherapy was based on gemcitabine (50 mg/m² administered bi-weekly).

\begin{tabular}{|c|c|c|c|}
\hline Organs & Mean & Minimum & Maximum \\
\hline Liver & 11.45 & 0.53 & 57.80 \\
\hline Stomach & 17.43 & 1.17 & 54.35 \\
\hline Right kidney & 8.67 & 1.57 & 32.20 \\
\hline Left kidney & 6.47 & 1.40 & 28.14 \\
\hline PTV1 (Tumor + LN) & 55.62 & 42.54 & 61.94 \\
\hline PTV2 (Tumor) & 59.82 & 53.99 & 62.19 \\
\hline
\end{tabular}

TABLE 1: Dose to organs at risk (Gy)

LN: lymph nodes. PTV: planning target volume. 
A physical examination and an evaluation of acute toxicity were performed on a weekly basis. After the treatment, patients were followed on a regular basis. The acute and late toxicities were evaluated according to common terminology criteria for adverse events (CTCAE) v3.0 during and after treatment. Overall and disease-free survival rates were calculated from the date of diagnosis. The median follow-up was 8.0 months (range 1-42 months). All 24 patients were evaluated for toxicity and survival.

\section{Results}

\section{Acute toxicities}

The majority of patients experienced grade 1 (G1) to grade 2 (G2) acute toxicity except for one patient who presented with severe digestive bleeding, potentially treatment-related. Grade 1 toxicities were observed in $58.3 \%$ of patients (14 out of 24 ) and grade 2 in $29.2 \%$ (seven out of 24 ), and consisted of fatigue, nausea, emesis, and weight loss. Grade 3 toxicities were experienced by $12.5 \%$ of patients (three out of 24) and consisted mainly of asthenia occurring at the end of the treatment.

\section{Late toxicities}

Late toxicities consisted mainly of G1 digestive toxicities. Grade 1 toxicities were observed in $83.3 \%$ (20 of 24 ) and G2 toxicities in $4.2 \%$ ( 1 of 24 ). Two patients died within three months after the completion of radiotherapy but without known toxicities. Three patients presented with digestive bleeding and two patients presented with an ileus. All these five later patients were known to have a loco-regional disease progression.

\section{Oncologic results}

The actuarial overall survival rates were $70 \%$ at 12 months, $39 \%$ at 24 months, and $8.6 \%$ at 36 months. The disease-free survival (DFS) rates were $48.8 \%$ at 12 months, $32.5 \%$ at 24 months, and $12.2 \%$ at 36 months. According to the tumor status, the DFS rates were of $83.3 \%$ and $41.7 \%$ at 12 and 36 months for R1 resected patients and $70 \%$ and $10 \%$ for non-operated patients. For all patients, the loco-regional control (LRC) rates were of $67.3 \%, 56.1 \%$, and $21.0 \%$ at 12,24 , and 36 months, respectively. More than $70 \%$ of the patients were deceased at the time of this analysis. Although the causes of death were sometimes difficult to determine (death at home, no autopsy), we noted that most of deaths were due to loco-regional or distant progression. Local progression consisted of stomach and duodenum invasion while distant progression consisted mainly of metastases to the liver, lungs, or the peritoneum.

\section{Discussion}

Treatment of patients with unresected or R1 resected pancreatic cancer remains a challenge. Without any treatment, the prognosis is very poor. The treatment may consist of a combined radiochemotherapy as described in the present study. Due to the retrospective nature of our study we are aware of the limitations and the caution that we should have in interpreting the results. Nevertheless, in line with some published data, the current study confirms the feasibility of high dose RT combined with gemcitabine-based chemotherapy with acceptable acute and late toxicities.

There is relatively less information on the behaviour of R1 resected patients treated with adjuvant radiotherapy and most of them come from sub-group analysis. Even though the number of patients is quite limited, the results showed in this study for R1 resected patients were slightly better than those of nonoperated patients.

At three years, Blackstock et al. [16] reported an overall survival (OS) rate of 5\% for patients with unresectable tumors treated by radiotherapy $(50.4 \mathrm{~Gy})$ and gemcitabine. Our results compare favorably with an OS of $8.6 \%$ at three years; however, our study includes $25 \%$ of R1 resected patients. On the other hand, the OS of R1 patients in our study is at least as good as the one reported in the radiation therapy oncology group (RTOG) 9704 study [23].

Willett et al. [24], observed a decreased death risk with adjuvant chemoradiotherapy in R0 resected patients compared to R1 patients with $29 \%$ vs $18 \%$ five-year OS. No patients with R1 margins survive beyond 41 months. This confirms the association of R1 margins with a worse outcome and also the relevance of considering adjuvant local and systemic treatment for these patients.

Rwigema et al. [25] reported that patients with R1 resected tumors benefit from adjuvant stereotactic external beam RT (SBRT), with a two-year OS rate of $61.4 \%$. This was higher than the two-year OS rate of 39\% for similar patients receiving adjuvant gemcitabine alone observed in the study of Oettle et al. [8]. A recent study by Chang et al. [26], showed results suggesting that patients with close surgical margins may benefit from more aggressive therapeutic approaches such as SBRT that target loco-regional disease.

Treatment of locally unresectable pancreatic cancer remains highly challenging. Among the attempts to improve the outcome, multimodal approaches such as radiotherapy plus chemotherapy have been regularly 
explored. Because of the risk toxicity particularly to gastrointestinal tracts, RT doses have been historically limited, delivered at best with a maximum dose of $50 \mathrm{~Gy}$ or with treatment interruption halfway through. This made the interpretation of the results quite difficult $[9,27,28]$. An early study of the Gastrointestinal Tumor Study Group (GITSG) attempted a dose escalation and compared 40 Gy with 60 Gy, combined with chemotherapy. No improvement in OS or in local control was seen in the higher dose arm. Here again the doses were increased but in courses of $20 \mathrm{~Gy}$ with two-week breaks. These RT schemes are known to be less effective than the continuous ones and may contribute to a loss in the treatment efficacy [29].

On the other hand Krishnan et al. [14] did an interesting study at the MD Anderson Cancer Center, by comparing the outcomes of patients with locally advanced pancreatic cancer (LAPC) who were treated with curative intent either by dose-escalated IMRT or by standard fractionation radiation therapy regimens to a median dose of $50.4 \mathrm{~Gy}$. Concurrent capecitabine was the main chemotherapy regimen used. They noticed that patients who received a dose with a biologically effective dose (BED) > 70 Gy had a superior OS and an improved recurrence-free survival (RFS). Interestingly, no additional toxicity in the high-dose group was observed. Higher dose (BED) was the only predictor of improved OS on multivariate analysis. They concluded that radiation dose escalation during consolidative chemoradiation therapy after induction chemotherapy for LAPC patients improves OS and loco-regional RFS. They also suggest that the choice of the concurrent chemotherapy regimen may have an impact on the tolerability; capecitabine being possibly better tolerated than gemcitabine, cisplatin/5-FU, or 5-FU/mitomycin C.

Based on the National Cancer Data Base (NCDB), Zhong et al. [30] compared the clinical outcomes of patients treated with either conventional fractionated RT (CFRT) or SBRT for locally advanced, nonmetastatic pancreatic adenocarcinoma. The two cohorts were not exactly identical-the patients in the SBRT cohort were older, had a lower rate of chemotherapy utilization, and a lower proportion of T4 tumors while the patients in the CFRT cohort had a larger proportion of positive-node status. The RT dose in the CFRT group was of $50.4 \mathrm{~Gy}$, while in the SBRT arm it was of $40 \mathrm{~Gy}$ (median dose per fraction of $8 \mathrm{~Gy}$ ). The two-year OS rate was significantly better in the SBRT group compared to CFRT group, with $20.3 \%$ versus $16.3 \%$. They didn't find any subgroup that appeared to significantly benefit from CFRT versus SBRT. However, in order to validate those first results a confirmation from randomized trials is warranted.

\section{Conclusions}

The present study confirms the feasibility of high dose RT combined with gemcitabine-based chemotherapy in patients with locally advanced pancreatic adenocarcinoma. While the outcome remains unsatisfactory in this disease, some patients seem to have benefited from this aggressive therapy that merits to be investigated further. The other emerging RT strategy, namely SBRT, showed encouraging results and merits future investigations too. The high rates of distant metastasis stress the need for more efficient systemic therapies.

\section{Additional Information \\ Disclosures}

Human subjects: Consent was obtained or waived by all participants in this study. Animal subjects: All authors have confirmed that this study did not involve animal subjects or tissue. Conflicts of interest: In compliance with the ICMJE uniform disclosure form, all authors declare the following: Payment/services info: All authors have declared that no financial support was received from any organization for the submitted work. Financial relationships: All authors have declared that they have no financial relationships at present or within the previous three years with any organizations that might have an interest in the submitted work. Other relationships: All authors have declared that there are no other relationships or activities that could appear to have influenced the submitted work.

\section{References}

1. Siegel RL, Miller KD, Jemal A: Cancer statistics, 2017. CA Cancer J Clin. 2017, 67:7-30. 10.3322/caac.21387

2. Jemal A, Siegel R, Ward E, Hao Y, Xu J, Thun MJ: Cancer Statistics, 2009. CA Cancer J Clin. 2009, 59:225-49. 10.3322/caac.20006

3. Parkin DM, Bray F, Ferlay J, Pisani P: Global cancer statistics, 2002. CA Cancer J Clin. 2005, 55:74-108. 10.3322/canjclin.55.2.74

4. Cubilla AL, Fortner J, Fitzgerald PJ: Lymph node involvement in carcinoma of the head of the pancreas area . Cancer. 1978, 41:880-7. 10.1002/1097-0142(197803)41:3<880::AID-CNCR2820410315>3.0.CO;2-j

5. Nagai H, Kuroda A, Morioka Y: Lymphatic and local spread of T1 and T2 pancreatic cancer. A study of autopsy material. Ann Surg. 1986, 204:65-71.

6. Strohl MP, Raigani S, Ammori JB, Hardacre J, Kim J: Surgery for localized pancreatic cancer: the trend is not improving. Pancreas. 2016, 45:687-93. 10.1097/MPA.0000000000000511

7. Murphy MM, Simons JP, Hill JS, et al.: Pancreatic resection: a key component to reducing racial disparities in pancreatic adenocarcinoma. Cancer. 2009, 115:3979-90. 10.1002/cncr.24433

8. Oettle H, Post S, Neuhaus P, et al.: Adjuvant chemotherapy with gemcitabine vs observation in patients undergoing curative-intent resection of pancreatic cancer: a randomized controlled trial. JAMA. 2007, 297:267-77. 10.1001/jama.297.3.267

9. Neoptolemos JP, Stocken DD, Friess H, et al.: A randomized trial of chemoradiotherapy and chemotherapy 
after resection of pancreatic cancer. N Engl J Med. 2004, 350:1200-10. 10.1056/NEJMoa032295

10. Hsu CC, Herman JM, Corsini MM, et al.: Adjuvant chemoradiation for pancreatic adenocarcinoma: the Johns Hopkins Hospital-Mayo Clinic collaborative study. Ann Surg Oncol. 2010, 17:981-90. 10.1245/s10434-0090743-7

11. Yeo CJ, Cameron JL, Lillemoe KD, et al.: Pancreaticoduodenectomy for cancer of the head of the pancreas. 201 patients. Ann Surg. 1995, 221:721-731. 10.1097/00000658-199506000-00011

12. Sener SF, Fremgen A, Menck HR, Winchester DP: Pancreatic cancer: a report of treatment and survival trends for 100,313 patients diagnosed from 1985-1995, using the National Cancer Database. J Am Coll Surg. 1999, 189:1-7. 10.1016/S1072-7515(99)00075-7

13. Katz MHG, Pisters PWT, Evans DB, et al.: Borderline resectable pancreatic cancer: the importance of this emerging stage of disease. J Am Coll Surg. 2008, 206:833-846. 10.1016/j.jamcollsurg.2007.12.020

14. Krishnan S, Chadha AS, Suh Y, et al.: Focal radiation therapy dose escalation improves overall survival in locally advanced pancreatic cancer patients receiving induction chemotherapy and consolidative chemoradiation. Int J Radiat Oncol. 2016, 94:755-65. 10.1016/j.ijrobp.2015.12.003

15. Krishnan S, Rana V, Janjan NA, et al.: Induction chemotherapy selects patients with locally advanced, unresectable pancreatic cancer for optimal benefit from consolidative chemoradiation therapy. Cancer. 2007, 110:47-55. 10.1002/cncr.22735

16. Blackstock AW, Tepper JE, Niedwiecki D, Hollis DR, Mayer RJ, Tempero MA: Cancer and leukemia group B (CALGB) 89805: phase II chemoradiation trial using gemcitabine in patients with locoregional adenocarcinoma of the pancreas. Int J Gastrointest Cancer. 2003, 34:107-16.

17. Crane CH, Ellis LM, Abbruzzese JL, et al.: Phase I trial evaluating the safety of bevacizumab with concurrent radiotherapy and capecitabine in locally advanced pancreatic cancer. J Clin Oncol. 2006, 24:1145-51. 10.1200/JCO.2005.03.6780

18. Li CP, Chao Y, Chi KH, et al.: Concurrent chemoradiotherapy treatment of locally advanced pancreatic cancer: gemcitabine versus 5-fluorouracil, a randomized controlled study. Int J Radiat Oncol Biol Phys. 2003, 57:98-104. 10.1016/S0360-3016(03)00435-8

19. Milano MT, Chmura SJ, Garofalo MC, et al.: Intensity-modulated radiotherapy in treatment of pancreatic and bile duct malignancies: toxicity and clinical outcome. Int J Radiat Oncol Biol Phy. 2004, 59:445-53. 10.1016/j.ijrobp.2003.11.003

20. Okusaka T, Ito Y, Ueno H, et al.: Phase II study of radiotherapy combined with gemcitabine for locally advanced pancreatic cancer. Br J Cance. 2004, 91:673-7. 10.1038/sj.bjc.6602001

21. Shinchi H, Takao S, Noma H, Matsuo Y, Mataki Y, Mori S, Aikou T: Length and quality of survival after external-beam radiotherapy with concurrent continuous 5-fluorouracil infusion for locally unresectable pancreatic cancer. Int J Radiat Oncol Biol Phys. 2002, 53:146-50. 10.1016/S0360-3016(01)02806-1

22. Von Hoff DD, Ervin T, Arena FP, et al.: Increased survival in pancreatic cancer with nab-paclitaxel plus gemcitabine. N Engl J Med. 2013, 369:1691-703. 10.1056/NEJMoa1304369

23. Abrams RA, Winter KA, Regine WF, et al.: Failure to adhere to protocol specified radiation therapy guidelines was associated with decreased survival in RTOG 9704--a phase III trial of adjuvant chemotherapy and chemoradiotherapy for patients with resected adenocarcinoma of the pancreas. Int J Radiat Oncol Biol Phys. 2012, 82:809-16. 10.1016/j.ijrobp.2010.11.039

24. Willett CG, Lewandrowski K, Warshaw AL, Efird J, Compton CC: Resection margins in carcinoma of the head of the pancreas. Implications for radiation therapy. Ann Surg. 1993, 217:144-8.

25. Rwigema JC, Parikh SD, Heron DE, et al.: Stereotactic body radiotherapy in the treatment of advanced adenocarcinoma of the pancreas. Am J Clin Oncol. 2011, 34:63-9. 10.1097/COC.0b013e3181d270b4

26. Chang DK, Johns AL, Merrett ND, et al.: Margin clearance and outcome in resected pancreatic cancer . J Clin Oncol Oncol. 2009, 27:2855-62. 10.1200/JCO.2008.20.5104

27. Kaiser MH, Ellenberg SS: Pancreatic cancer. Adjuvant combined radiation and chemotherapy following curative resection. Arch Surg. 1985, 120:899-903. 10.1001/archsurg.1985.01390320023003

28. Klinkenbijl JH, Jeekel J, Sahmoud T, et al.: Adjuvant radiotherapy and 5-fluorouracil after curative resection of cancer of the pancreas and periampullary region: phase III trial of the EORTC gastrointestinal tract cancer cooperative group. Ann Surg. 1999, 230:776-784.

29. Moertel CG, Frytak S, Hahn RG, et al.: Therapy of locally unresectable pancreatic carcinoma: a randomized comparison of high dose (6000 rads) radiation alone, moderate dose radiation (4000 rads +5 -fluorouracil), and high dose radiation + 5-fluorouracil: the Gastrointestinal Tumor Study Group. Cancer. 1981, 48:170510. 10.1002/1097-0142(19811015)48:8<1705::AID-CNCR2820480803>3.0.CO;2-4

30. Zhong J, Patel K, Switchenko J, et al.: Outcomes for patients with locally advanced pancreatic adenocarcinoma treated with stereotactic body radiation therapy versus conventionally fractionated radiation. Cancer. 2017, 123:3486-3493. 10.1002/cncr.30706 